\title{
Public Health Problems in Bangladesh: Issues and challenges
}

\author{
Faisal Muhammad ${ }^{2}$, Moniruddin Chowdhury ${ }^{2}$, Mohd Arifuzzaman $^{3}$, ABM Alauddin Chowdhury $^{4}$
}

${ }^{1}$ Research Assistant; ${ }^{3}$ Senior Coordination Officer; ${ }^{4}$ Assistant Professor; Department of Public Health, Daffodil International University, Dhaka, Bangladesh. ${ }^{2}$ Assistant Professor, Department of Medicine and Health Sciences, University Tunku Abdul Rahman, Sungai Long Campus, Kajang, Selangor D.E, Malaysia

\section{Abstract}

In South-East Asia the main public health issues are infectious diseases and communicable diseases. Public health has improved markedly in Bangladesh over the past three decades. Nevertheless, Bangladesh faces major health challenges. A scoping study was performed according to York methodology. The study was aimed to find out the major public health issues and challenges in Bangladesh. Bangladesh has one of the worst burdens of childhood malnutrition in the world. Communicable diseases are a major cause of death and disability in Bangladesh. Unsafe food remains a major threat to public health each year, citizens suffer from the acute effects of food contaminated by microbial pathogens, chemical substances and toxins. Bangladesh still ranks among the top ten countries in the world with the highest TB burden. Pneumonia and other infections are major causes of death among young children. In Bangladesh only $1 \%$ of the population is reported to be HIV-positive, but rates are much higher among high-risk populations: injecting drug users, sex workers, and men who have sex with men. The toll of non-communicable diseases - chronic diseases, cancer, diabetes, cardiovascular diseases, and chronic respiratory diseases - is increasing in Bangladesh as the population becomes more urbanized. The converging pressures of global climate change and urbanization have a devastating effect on Bangladesh's most vulnerable populations. The disease burden Bangladesh is further exacerbated by unsanitary living conditions that underscore the poor economic conditions of both urban and rural home dwellers. There are still several issues that Bangladesh health care system is yet to tackle, governance, accessibility, and affordability are key issues that are preventing the implementation of solutions to the public health issues in Bangladesh.

Keywords: Public-health, Infectious disease, Communicable diseases, NCD, Bangladesh.

\section{Introduction}

Bangladesh, with a population of 1.55 million in 2012, is one of the most densely populated countries in the world, having a population density of 1050 per $\mathrm{km}^{2}$. The male/ female ratio is $104.9 / 100$ and the annual population growth rate is $1.37 \% .^{2}$ The population of Bangladesh is very young as depicted by its wide-based population pyramid. A large cohort of the young population will enter reproductive age in the coming decades, a phenomenon partly explaining why the adolescent (15-19 years) fertility rate in Bangladesh of 118 per 1000 women and increasing life expectancy at birth of 69 years in $2011 .^{3}$

Bangladesh Demographic and Health Survey is not expected to decrease significantly for decades. As in other countries, the population is ageing over time due to decreasing fertility rates (6.3 births per woman in 1975 to 2.3 in 2011). ${ }^{3}$

Unsafe food remains a major threat to public health ${ }^{4}$ each year, citizens suffer from the acute effects of food contaminated by microbial pathogens, chemical substances and toxins. There is a need to minimize the consumer's exposure to unhygienic, contaminated and adulterated food and drinks through strict laws to control marketing of such products. ${ }^{1}$

One such factor is violence against women. This is a widespread social problem that causes mental stress, physical suffering and even death, and is believed to be

\section{Practice Points}

- Bangladesh is one of the most densely populated countries in the world, having a population density of 1050 per $\mathrm{km}^{2}$.

- Bangladesh still ranks among the top ten countries in the world with the highest TB burden.

- Pneumonia and other infections are major causes of death among young children.

- In Bangladesh only $1 \%$ of the population is reported to be HIV-positive, but rates are much higher among high-risk populations: injecting drug users, sex workers, and men who have sex with men.

- Improving health care-seeking behavior such as education, awareness-raising and skills building in recognizing and treating pneumonia and obstetric emergencies.

grossly underreported. One study reveals that in Bangladesh about $52 \%$ of men in both urban and rural sites reported ever physically assaulting female intimate partners. ${ }^{5}$ According to the Joint Monitoring Programme (JMP) of the World Health Organization (WHO) and the United Nations Children's Fund (UNICEF), $83 \%$ of the population have access to safe water for drinking. ${ }^{6}$ While the population at risk of

Correspondence: Dr. ABM Alauddin Chowdhury, Assistant Professor, Department of Public Health, Faculty of Allied Health Science, Daffodil International University, Dhaka, Bangladesh. Email: dralauddin@daffodilvarsity.edu.bd.

South East Asia Journal of Public Health 2016;6(2):11-16. (C) 2016 Muhammad et al., publisher and licensee Public Health Foundation Bangladesh. This is an Open Access article which permits unrestricted non-commercial use, provided the original work is properly cited. 
consuming arsenic contaminated drinking water is estimated at 20 million, the number of exposed persons may well be lower because of ongoing awarenessraising activities. ${ }^{1,7}$ The challenge is to ensure access to safe water for $100 \%$ of the population. Basic sanitation coverage is $55 \%$ against the target of $70 \%$ by $2015 .{ }^{6}$ Although more than 90 million people in Bangladesh shifted to fixed-point defecation in the last five years, diarrheal diseases remain a leading cause of child and infant morbidity. A research study shows that only $1 \%$ of the population wash their hands with soap and water before having a meal, $0.7 \%$ before feeding children, and $30 \%$ after defecation. ${ }^{8}$

Behavior change through hygiene promotion is a priority to achieve the health benefit of sanitation coverage. The issue of total sanitation coverage also demands a concept that goes beyond excreta disposal to include the environmental sanitation issues associated with the safe management of solid waste, household wastewater and storm water. Waste management, including clinical waste, solid waste, domestic and industrial wastewater, is putting substantial burden on the environment and creating public health risks. ${ }^{8}$ Management of clinical waste including sharps in facilities and elsewhere is a challenge that has to be immediately addressed. Environmental pressures, exacerbated by climate change, remain significant and could easily worsen if remedial actions at the local and global levels are not taken. While the population is expected to stabilize at around 200 million, growing wealth and mass population movements will place further enormous strains on ecosystems and the living environment. ${ }^{1}$

The major communicable diseases in Bangladesh are vaccine-preventable diseases (VPD), tuberculosis, malaria HIV/AIDS and neglected tropical diseases (Leprosy, Kala-azar, Lymphatic filariasis and dengue). However the disease burden in Bangladesh has shifted from communicable to non-communicable diseases (NCDs) like cardiovascular diseases, diabetes, cancer, and chronic respiratory diseases. More than half of hospital deaths are due to NCDs. Data from the Matlab demographic survey site showed an increasing trend of NCD deaths from 1986 to 2006 especially due to cardiovascular diseases. ${ }^{9}$ The study was aimed to find out the major public health issues and challenges in Bangladesh.

\section{Materials and methods}

A review was performed according to York methodology, outlined by Arksey and O'Malley, 2005 from the University of York, United Kingdom. ${ }^{10}$ Published academic articles, World Health Organizations (WHO) reports, Millennium development Goals (MDGs) reports, and the Handbook for Journalist in Bangladesh ${ }^{11}$ (in association with Johns Hopkins Bloomberg School of Public Health,Center for Communication Programs, Measure DHS and USAID, August 2013), were used for comprehensive search.

Many related papers were reviewed, during the review some research papers that met the criteria for the research question were included. The inclusion criterion was information about public health issues in Bangladesh; more recent research studies were used (2008 onwards). Papers were excluded if the study was not based on public health problems of Bangladesh. The review was done through reading the previous publications those found to meet the inclusion criteria were selected and finally all results were summarized and reported.

\section{Results and Discussion}

Bangladesh faces a number of health challenges which can be grouped as follows:

- Population problems

- Communicable diseases problems

- Nutritional problems

- Environmental sanitation problems

- Health problems

\section{Population problems}

Bangladesh has a population of more than 1.55 million people in 2012, is one of the most densely populated countries in the world, having a population density of 1050 per $\mathrm{km}^{2}$. 1,12 The male/female ratio is $104.9 / 100.0$ and the annual population growth rate is $1.37 \%{ }^{2}$ The population of Bangladesh is very young as depicted by its wide-based population pyramid. A large cohort of the young population will enter reproductive age in the coming decades, a phenomenon partly explaining why the adolescent (15-19) fertility rate in Bangladesh of 118 per 1000 women is not expected to decrease significantly for decades. ${ }^{3}$ As in other countries, the population is ageing over time due to decreasing fertility rates (6.3 births per woman in 1975 to 2.3 in 2011) and increasing life expectancy at birth of 69 years in $2011 .^{3}$ The national population is projected to grow to between 200 to 225 million over the next four decades. ${ }^{3}$ While fertility has declined, women have on average 2.3 children, and only about half use modern and effective contraceptive methods. ${ }^{3}$

Despite improvements in maternal health, Bangladesh still ranks in the bottom fourth of countries worldwide with approximately 240 deaths per 100,000 live births. ${ }^{11}$ Only one-in-four births takes place in a health care facility, putting both mothers and babies at risk. ${ }^{11}$

\section{Communicable diseases}

Communicable diseases are a major cause of death and disability in Bangladesh. While the prevalence of tuberculosis (TB) has declined substantially, Bangladesh still ranks among the top ten countries in the world with the highest TB burden. ${ }^{13}$ Bangladesh is one of the 22 high tuberculosis-burden countries. The incidence, prevalence and mortality estimates for tuberculosis (TB) are 225/100 000, 434 per 100000 and $45 / 100000$ respectively and $1.4 \%$ statistics of multidrug resistant cases. ${ }^{14}$ TB services are integrated in the primary health care system. The treatment success rate is $92 \%$ for the cohort of patients registered in $2011 .^{13}$

Pneumonia and water-borne diseases also are widely prevalent. Pneumonia and other infections are major causes of death among young children. ${ }^{11}$ Pneumonia is the leading cause of death worldwide in children under five years of age. According to the World Health Organization, nearly 400 children die each day from ARIs in Bangladesh. Pneumonia, infection, and birth asphyxia are major causes of under-five deaths in the country. Early detection and treatment of infection is key to saving lives. ${ }^{1}$ 
The human immunodeficiency virus (HIV), In Bangladesh, only $1 \%$ of the population is reported to be HIV-positive, but rates are much higher among highrisk populations: injecting drug users, sex workers, and men who have sex with men. The majority of Bangladeshi adults are not well informed about transmission or prevention of HIV. ${ }^{1}$ Bangladesh still has a low prevalence of $<1 \%$ among population groups most at risk. According to the latest serological surveillance, HIV prevalence among specific risk groups (people who use drugs, female and male sex workers, men who have sex with men, and Hijras) was reported to be $0.7 \% .{ }^{15}$ Bangladesh has performed well in halting communicable diseases. ${ }^{16}$ Available data show that the prevalence of HIV/AIDS in Bangladesh currently low and thus is still below an epidemic level. According to National AIDS/STD Programmes (NASP), condom use rate at last high risk sex was $43.33 \%$ in 2013. According to National AIDS/STD Programmes (NASP), proportion of population aged 1524 years with comprehensive correct knowledge of HIV/AIDS is $17.7 \%$ in $2013 .{ }^{16}$

Malaria is common in some areas in northeast and southeast Bangladesh. Out of the total 64 districts, 13 are high endemic areas of malaria transmission. A total of 50,000 confirmed malaria cases are reported each year, but under-reporting is widespread. Prevention is key: sleeping under an insecticide-treated bed net every night and wearing long sleeved light colored clothing all help prevent mosquito bites. ${ }^{1}$ Malaria is a major public health problem in Bangladesh with 13.2 million people at risk. Malaria is highly endemic in 13 of the country's 64 districts, from where more than $95 \%$ of the total malaria cases are reported. ${ }^{17}$ Dengue is a mosquitoborne viral infection that causes flulike symptoms and occasionally develops into a potentially fatal infection. As opposed to malaria, dengue is carried in mosquitoes that are active during daylight hours. Approximately half of the world's population is at risk, largely in urban areas in tropical and sub-tropical regions, and global incidence has grown markedly in recent decades. There is no treatment for dengue, however early detection and access to medical care lowers fatality rates.

\section{Nutritional problems}

Poor nutrition, often called under-nutrition can damage physical, intellectual, and mental health, leading to reduced immunity, increased susceptibility to disease, impaired physical and mental development and reduced educational and economic productivity. It is well recognized that the period from birth to two years of age is the critical window of behavioral and cognitive development promotion. In Bangladesh, close to $50 \%$ of children-under-five are stunted due to poor nutrition, with urban poor most affected. ${ }^{3}$ Twenty-two percent of infants are low birth weight, only $43 \%$ of infants are exclusively breastfed, and $41 \%$ of children-under-five are moderately to severely underweight. Improving nutrition should be a major public health priority in Bangladesh. ${ }^{3}$ Although infant and child mortality is decreasing, poor nutrition is a critical health problem in Bangladesh. ${ }^{11}$ About half of children age 6-59 months suffers from anemia; four-in-ten are stunted; and one in three is underweight. ${ }^{11}$ Bangladesh has one of the worst burdens of childhood malnutrition in the world. ${ }^{6}$

\section{Environmental sanitation problems}

Around 1.1 billion people globally do not have access to improved water supply sources and 2.4 billion people do not have access to any type of improved sanitation facility. Approximately 2 million people die every year due to diarrheal diseases, the most vulnerable of which are children under 5 years of age. The most affected are the populations in developing countries, living in extreme conditions of poverty, normally peri -urban dwellers or rural inhabitants. Sanitation generally refers to the toilets or latrines for safe disposal of human urine and feces. Inadequate sanitation is a major cause of disease worldwide. Improving sanitation is a proven public health intervention at the household, community, and national levels. The word 'sanitation' also refers to the safe disposal of environmental waste, such as garbage and wastewater. Hygiene refers to conditions and practices that help to maintain health and prevent the spread of diseases.

Medical hygiene includes a specific set of practices, for example environmental cleaning, infection control in health care facilities, hand hygiene, water and sanitation, and safe disposal of medical waste. ${ }^{3}$ According to the Joint Monitoring Programme (JMP) of WHO and the United Nations Children's Fund (UNICEF), $83 \%$ of the population have access to safe water for drinking. ${ }^{6}$ While the population at risk of consuming arsenic contaminated drinking water is estimated at 20 million, the number of exposed persons may well be lower because of ongoing awareness-raising activities. ${ }^{6}$ The challenge is to ensure access to safe water for $100 \%$ of the population. Basic sanitation coverage is $55 \%$ against the target of $70 \%$ by $2015 .^{6}$ Although more than 90 million people in Bangladesh shifted to fixed-point defecation in the last five years, diarrheal diseases remain a leading cause of child and infant morbidity. A research study shows that only $1 \%$ of the population wash their hands with soap and water before having a meal, $0.7 \%$ before feeding children, and $30 \%$ after defecation. $^{8}$ Behavior change through hygiene promotion is a priority to achieve the health benefit of sanitation coverage. The issue of total sanitation coverage also demands a concept that goes beyond excreta disposal to include the environmental sanitation issues associated with the safe management of solid waste, household wastewater and storm water.

Waste management, including clinical waste, solid waste, domestic and industrial wastewater, is putting substantial burden on the environment and creating public health risks. Management of clinical waste including sharps in facilities and elsewhere is a challenge that has to be immediately addressed. Environmental pressures, exacerbated by climate change, remain significant and could easily worsen if remedial actions at the local and global levels are not taken. While the population is expected to stabilize at around 200 million, growing wealth and mass population movements will place further enormous strains on ecosystems and the living environment. ${ }^{5}$ Concrete and concerted multi sector programs on adapting to climate change need to be in place.

Health problems in Bangladesh

The major public health issues and problems in Bangladesh include acute respiratory infections (ARIs) and pneumonia, dengue, diarrhea and water- 
borne diseases, essential nutrition, HIV/AID, improved water, sanitation and hygiene (WASH), malaria, maternal, newborn and child health (MNCH), neglected tropical disease, non-communicable disease (NCDs), road safety, tuberculosis, vaccination and immunization. 10

Diarrheal disease is the second leading cause of death in children under five years old, and is responsible for killing around 760000 children every year. Diarrhoea can last several days, and can leave the body without the water and salts that are necessary for survival. Most people who die from diarrhoea actually die from severe dehydration and fluid loss. Children who are malnourished or have impaired immunity as well as people living with HIV are most at risk of life-threatening diarrhoea. ${ }^{18}$ Measures to prevent childhood diarrheal episodes include: promoting exclusive breastfeeding, improving hygiene and sanitation, increasing access to improved sources of drinking water and sanitation facilities, zinc intake to ensure intestinal health, and hand washing with soap at critical times throughout the day (after using the bathroom, changing a diaper, before preparing a meal or eating). Treatment of childhood diarrhea has improved in Bangladesh. According to the 2011 Bangladesh DHS, diarrhea is no longer a leading cause of death among children. ${ }^{3}$

Ensuring optimal health for girls of reproductive age, improving the health and nutrition of mothers-to-be, and providing quality reproductive health services including ante- and post-natal care are pivotal to ensuring safe motherhood. Improving the health of mothers-to-be, including provision of quality reproductive health and family planning services, is pivotal to addressing many underlying causes of maternal and child mortality. A vast majority of maternal deaths are caused by direct obstetric causes such as hemorrhage, infection, hypertensive disorders of pregnancy such as eclampsia, and complications from unsafe abortion. For every woman who dies from complications related to childbirth, approximately 20 more suffer injuries, infections and disabilities that may be left untreated and ignored, resulting in life-long pain and social and economic exclusion. Many maternal and newborn deaths can be prevented through identification of complications and timely care, particularly in the first $24-48$ hours after giving birth. Women who receive routine antenatal care, optimal nutrition and practice birth spacing are at a healthy advantage. Bangladesh's maternal mortality ratio has declined over time. However, with 240 deaths for every 100,000 live births, Bangladesh ranks well behind most other large Asian countries. ${ }^{7}$ Bangladeshi women are less likely to receive antenatal care and to give birth in health care facilities than women in many other countries, increasing risks to mothers and babies.

Neglected Tropical Disease; Such as Leishmaniasisis (Kala-azar) is caused by a parasite transmitted by sand flies that often live in mud walls of homes. The disease is usually found among the poorest populations and is most prevalent in the northwestern part of the country. A new oral drug, Miltefosine, can now cure the disease however a counterfeit, substandard generic is often available in through the nationwide treatment program. Local drug regulations and supply monitoring must happen in order to eradicate this disease. Lymphatic filariasis ("elephantiasis") is caused by infection with roundworms after transmission via mosquito bite. Adult worms lodge in human lymph nodes and disrupt the immune system. The disease causes local infections, tremendous swelling of lymph nodes, disfiguration and pain. Inexpensive medicines can cure the disease, and mass single-dose drug administration campaigns are run to help eliminate the disease in at -risk endemic communities. Leishmaniasisis and lymphatic filariasis occur disproportionately in Bangladesh. Ninety percent of visceral leishmaniasis cases occur in India, Bangladesh, Nepal, Sudan, Ethiopia and Brazil. In 2010, there were 3,800 cases of visceral leishmaniasis reported in Bangladesh alone out of a global total of approximately 1.5 million. Approximately 20 million people in Bangladesh suffer from lymphatic filariasis yet closer to 70 million people are at risk of infection as it is highly endemic in 33 out of 64 districts. It is considered a major public health issue in country. ${ }^{1}$

Non-communicable diseases (NCD), defined as medical conditions that cannot be transmitted from person-to-person, are a growing public health problem in Bangladesh as well as in the rest of the world. WHO report in 2002 identified unhealthy diet, physical inactivity, tobacco use, harmful use of alcohol, overweight, raised blood pressure, raised total cholesterol levels and raised blood glucose as the most prevalent NCD risk factors among the world population. ${ }^{19}$ The toll of non-communicable diseases - chronic diseases, cancer, diabetes, cardiovascular diseases, and chronic respiratory diseases - is increasing in Bangladesh as the population becomes more urbanized. ${ }^{15}$ In the First National Survey to measure blood pressure and blood glucose, about one in three women and about one in five men age 35 and older has elevated blood pressure and roughly one in ten has elevated blood glucose, an indication of diabetes. ${ }^{11}$ Cancer is the sixth leading cause of death in Bangladesh, accounting for more than 150,000 deaths annually. $^{20}$ Major non-communicable diseases include high blood pressure, diabetes, cancer and asthma. Cardiovascular (heart) disease is now considered to be a leading cause of death in Bangladesh. About one in three women and about one in five men age 35 and older has elevated blood pressure and roughly one in ten women and men age 35 and older has elevated blood glucose, an indication of diabetes. ${ }^{20}$ More than 4,000 people die each year on Bangladesh's roads. According to the World Health Organization, road traffic injuries cause a loss of about 2\% of GDP in Bangladesh each year - an amount nearly equal to the total foreign aid the country receives. Road accidents have many causes: unskilled and reckless drivers, speeding, overloading, vehicle defect, hazardous road conditions, poor supervision or adherence to traffic rules. Because of the sheer number of roadrelated accidents and mortality, road safety should be considered a public health issue when reporting on Bangladesh. ${ }^{11}$ Immunization makes a person is resistant to an infectious disease, typically by the administration of a vaccine. According to the 2011 Bangladesh DHS, $86 \%$ of children aged 12-23 months have received all recommended vaccinations. Immunization is a proven tool for controlling and eliminating life-threatening infectious diseases and is estimated to avert 
between 2 and 3 million deaths each year. It is one of the most cost-effective health investments, with proven strategies that make it accessible to even the most hardto reach and vulnerable populations. Vaccination has clearly defined target groups; it can be delivered effectively through outreach activities; and does not require any major lifestyle change. ${ }^{1,3}$ Unsafe food remains a major threat to public health. ${ }^{4}$ Each year, citizens suffer from the acute effects of food contaminated by microbial pathogens, chemical substances and toxins. There is a need to minimize the consumer's exposure to unhygienic, contaminated and adulterated food and drinks through strict laws to control marketing of such products. ${ }^{1}$ Improving women's access to quality health services and addressing the underlying sociocultural factors that prevent it are major challenges. One such factor is violence against women. This is a widespread social problem that causes mental stress, physical suffering and even death, and is believed to be grossly underreported. One study reveals that in Bangladesh about $52 \%$ of men in both urban and rural sites reported ever physically assaulting female intimate partners. ${ }^{5}$

\section{Conclusion}

There are still several issues that Bangladesh health care system is yet to tackle; governance, accessibility, and affordability are key issues that are preventing the implementation of solutions to the public health issues in Bangladesh. ICDDRB, WHO, and several other global organizations are making attempt to aid Bangladesh in resolving its health problems. The converging pressures of global climate change and urbanization have a devastating effect on Bangladesh's most vulnerable populations. At this moment, millions of people in the country are exposed to extremely high food and water-borne disease risks such as bacterial and protozoal diarrhea, Hepatitis A and E, and typhoid fever. The disease burden Bangladesh is further exacerbated by unsanitary living conditions that underscore the poor economic conditions of both urban and rural home dwellers. Sadly, these poor health outcomes disproportionately impact children under the age of five years, causing widespread morbidity and mortality; the second leading cause of death in children under five is diarrheal disease.

The study put forward following recommendations to improve the standard of public health in Bangladesh:

- Improving health care-seeking behavior such as education, awareness-raising and skills building in recognizing and treating pneumonia and obstetric emergencies.

- Rapid urbanization which creates conditions that make slum dwellers vulnerable to disease as a result of problems of sanitation, hygiene and the supply of clean water.

- The emergence of arsenic in tube well water, leading to arsenic poisoning, solution measures has to be taken by those that held the responsibility (i.e. Government).

- Reduce the burden of communicable diseases, including vaccine-preventable diseases, tuberculosis, malaria, HIV/AIDS, and neglected tropical diseases by sustaining high immunization coverage and introduce new vaccines; achieve "universal access" to quality diagnosis and treatment for all tuberculosis patients; strengthen diagnosis and treatment of malaria, addressing cross-border transmission; build capacity of the National AIDS/STD (sexually transmitted disease) Programme for effective treatment, care and support to HIVpositive people; strengthen diagnosis and treatment of kala-azar, filariasis, leprosy and dengue.

- Reduce the burden of non-communicable diseases through health promotion, risk reduction and cost effective management.

- Reduce health, nutrition, environmental and occupational risk factors throughout the life course by supporting the development, adaptation and use of national policies, guidelines, standards, and tools to enhance health services for mothers and children from birth to adolescence; build the capacity of health providers to ensure quality services; contribute to the development of a functioning result-based Programme, monitoring and evaluation system on reproductive, maternal and child health at the national level; support the national strategy to mainstream nutrition through the development of policy, protocols and guidelines involving different sectors; build capacity of the local government on safe water by integrating the Water and Sanitation Programme into water supply management and building climate-resilient water, sanitation and hygiene (WASH).

- Promote universal health coverage with strengthened health systems based on primary health.

- Reduce mortality, morbidity and societal disruption resulting from epidemics, natural disasters, conflicts, environmental, and foodrelated emergencies, through prevention, preparedness, response and recovery activities that build resilience and use a multi sectoral approach.

\section{Competing interest}

The authors declare that they have no competing interests.

\section{Acknowledgements}

The authors would like to thank the Public Health Department of Daffodil International University, Dhaka, Bangladesh for advice, assistance and support throughout this research work.

\section{References}

1. World Health Organization. Bangladesh country data. http://www.who.int/gho/countries/en/ (accessed Dec 2016)

2. Ministry of Planning. Sample vital registration system report. Dhaka: Bangladesh Bureau of Statistics, 2011. 
3. National Institute of Population Research and Training. Bangladesh demographic and health survey. Dhaka: NIPORT, 2011.

4. Rahman S, Majumder MAA, Rabiul A, Moslehuddin SM, Das P, Rahman N. The extent and magnitude of formalin adulteration in fish sold in domestic markets of Bangladesh: A literature review. Int $J$ of Consum Stud 2016; 40 (2);152-9

5. United Nations Population Fund (UNPFA). Study report on men's attitude and practices regarding gender and violence against women in Bangladesh. Dhaka: UNFPA, 2011.

6. World Health Organization (WHO), United Nations Children's Fund (UNPFA). Joint monitoring Programme for water supply and sanitation, country file Bangladesh 2013 update. Dhaka: WHO \& UNICEF, 2013.

7. Flanagan SV, Johnston RB, Zheng Y. Arsenic in tube well water in Bangladesh: health and economic impacts and implications for arsenic mitigation. Bull World Health Orga 2012; 90:839 $-46$.

8. Johnston RB, Halder AK, Huda Md TN, Akhter $\mathrm{S}$, Mahmood Al A, Huque R, et al. Monitoring impacts of WASH interventions: the case of SHEWAB 34th WEDC international conference. Addis Ababa, 2009.

9. Karar ZA, Alam N, Streat field PK. Epidemiologic transition in rural Bangladesh, 1986-2006. Global Health Action 2009.

10. Arksey H, O’Malley L. Scoping studies: towards a methodological framework. Int $J$ Soc Res Methodol 2005; 8(1), 19-32.

11. Writing About Health: A Handbook for Journalist in Bangladesh in association with Johns Hopkins Bloomberg School of Public Health Center for Communication Programs, Measure DHS and USAID. https:// www.k4health.org/toolkits/bangladesh-healthjournalists/overview-health-bangladesh (accessed Dec 2016)
12. Ministry of Health and Family Welfare. Health population and nutrition sector development program (HPNSDP) 2012: summary version of the health sector programme 2011-2016. Dhaka: MOH\&FW, 2012.

13. Ministry of Health and Family Welfare. National TB control programme. Annual report 2010. Dhaka: Directorate General of Health Services, 2010.

14. World Health Organization. Global Tuberculosis Report 2013. Geneva: WHO, 2013.

15. Ministry of Health and Family Welfare. National HIV Serological Surveillance 2011. Dhaka: Directorate General of Health Services, 2011.

16. Millennium Development Goals (MDGs). Bangladesh Progress Report 2015. Dhaka: Bangladesh Planning Commission, 2015.

17. Center for Disease Control and Prevention (CDC). Annual Report Book 2013. Atlanta: CDC, 2013.

18. World Health Organization (WHO). Diarrheal disease, Fact sheet. http://www.who.int/ mediacentre/factsheets/fs330/en/ (accessed Dec 2016)

19. World Health Organization (WHO). World Health Report 2002: Reducing risks, promoting healthy life. Geneva: WHO, 2003.

20. World Health Organization (WHO). Noncommunicable disease risk factor survey. Dhaka: WHO, 2010. 